\title{
PRIMARY SPLENIC LYMPHOMA: CAN TRUCUT BIOPSY AND R-CHOP SUPERSEDE SPLENECTOMY?
}

\section{Medicine}

\begin{tabular}{ll} 
Aanchal Sawhney & Junior resident, Rajiv Gandhi cancer institute, New Delhi. \\
\hline Mridusmita & $\begin{array}{l}\text { Senior Resident, Department of internal medicine, Sir Ganga Ram Hospital, New Delhi. } \\
\text { Khataniar* }\end{array}$ \\
\hline *Corresponding Author
\end{tabular}

Roopal Verma

Senior Resident, Department of internal medicine, Sir Ganga Ram Hospital, New Delhi.

\section{ABSTRACT}

Primary Splenic lymphoma is a rare type of Non Hodgkin's lymphoma of the spleen with or without splenic hilar lymph nodes with preservation of other organ systems. In this case, we encountered a patient who presented with fever of unknown origin since 25 days and was diagnosed with Primary splenic lymphoma based on a tru cut biopsy which was then confirmed by immunohistochemistry. Patient was treated with Chemotherapy alone and showed no recurrence after 6 months of follow up. The aim of reporting this case is to have a high index of suspicion for rare malignancies like PSL in cases of FUO. Contrary to the current guidelines, use of trucut biopsy can be used as a promising investigation to diagnose PSL and treatment with chemotherapy with R-CHOP regimen alone prevented recurrence of the tumour. Investigations like PET-CT which have $100 \%$ accuracy in diagnosing PSL can reduce the number of operative procedures like splenectomy used for its diagnosis and treatment.

\section{KEYWORDS}

Primary splenic lymphoma, Tru cut biopsy, R-CHOP regimen, FUO

\section{INTRODUCTION}

According to Das Gupta et al Splenic lymphoma is a lymphoma involving only the spleen and the splenic hilar lymph nodes presenting with an isolated splenomegaly in the absence of any other localized tumors, particularly in the liver or the para-aortic or mesenteric lymph nodes with absence of recurrence till 6 months post splenectomy(1) (2). Primary Splenic Lymphomas (PSL) is an extranodal lymphoma which comprises $2 \%$ of all Non-Hodgkin Lymphomas (3)and has an overall incidence of $<1 \%$. Most of the splenic lymphomas are of $\mathrm{B}$ cell origin. (3) The low incidence and limited reporting of this primary splenic diffuse large B cell lymphoma could be owed to the strict diagnostic definitions noted.

PSL can be categorized as a splenic lymphoma with circulating villous lymphocytes and marginal zone splenic lymphoma originating from a splenic B-cell structure separated by the mantle zone. (4)

The patient can be asymptomatic or can present with nonspecific symptoms like fever of unknown origin, weight loss and abdominal pain or abdominal fullness.(5) A hematological parameter like bicytopenia along with raised ESR and CRP are also noted in PSL.(6)

Conventionally, splenectomy and pathological examination of the specimen was the treatment of choice but various underexplored investigation options exist. Although PSL is best recognized on CECT, PET-CT has an accuracy of $100 \%$ in diagnosing PSL as it also rules out involvement of other organs which is essential in diagnosis of PSL(7). Also investigation modality like FDG-PET CT eliminates unnecessary splenectomies in patients with splenomegaly due to causes other than lymphomas. Core biopsy using a 21/22 gauge needle can determine the type of PSL.(8)

Splenectomy has been accepted as the primary management of choice as it eliminates the local response and prevents the neoplastic dissemination from its primary site.(8) In inoperable cases, Chemotherapy with Rituximab, Cyclophosphamide, Doxorubicin, Vincristin, Prednisone (R-CHOP) regimen and radiotherapy have also been proven as effective treatment modalities questioning the role of invasive procedures like splenectomy for the diagnosis and treatment of PSL.(8) The patient has provided written informed consent for the publication of her case information and clinical images.

\section{CASE REPORT}

A 62 years old female presented with a low grade fever for the past 25 days and altered sensorium for 2 days.Patient had a history of loss of appetite and abdominal fullness but no history of loss of weight or night sweats. There was no history of cough,pain abdomen,burning micturation or any gastrointestinal symptoms. She was a known case of Diabetes and Hypertension since 10 years. She was a non alcoholic and non smoker.

On clinical examination, she was conscious, disoriented with a Glasgow Coma Scale of 13/15. She was febrile (temperature- 101 F) with Blood pressure of $140 / 80 \mathrm{~mm} \mathrm{Hg}$. She was pale with no lymphadenopathy or pedal edema. Systemic Examination revealed mild hepatosplenomegaly. Other systems were normal.

Lab investigations showed anaemia (Hb-7.2g/dl), thrombocytopenia (Platelet-90,000/mm 3), raised ESR , hypoalbuminemia, and hyponatremia. Renal profile was normal.

Patient was initially admitted in the ICU and started on empirical antibiotics, metabolic derangements were corrected and other supportive measures were given. Gradually her sensorium improved.

An array of blood tests like Malarial antigen, Widal Test, Scrub Typhus $\mathrm{IgM} / \mathrm{IgG}, \mathrm{HIV}$ were done but were inconclusive. Chest X ray and 2D ECHO were within normal limits. Blood and urine culture were sterile. ANA was negative. CECT chest and abdomen was done which showed hepatosplenomegaly and a homogenous opacity of $2.5 \mathrm{X} 2 \mathrm{~cm}$ size in spleen. The MRI brain was not very significant and revealed old haemorrhages.

A CSF examination was done in view of altered sensorium and fever which showed high protein with normal cell count. CSF cytology was negative and culture was sterile.

Bone marrow aspiration and biopsy was done which revealed a reactive marrow.

As all the above investigations were non conclusive the patient was taken up for PET CT which showed FDG uptake by multiple ill defined lesions in the spleen; no other lymph nodes were involved as seen in Figure 1. Following this report, tissue sampling was planned. Trucut biopsy of spleen revealed diffuse large B cell Non Hodgkin's Lymphoma as seen in Figure 2, positive for CD20, PAX5, and CD79a. The Ki67 proliferative index was $80-90 \%$.

As a definitive treatment, Splenectomy was advised but was deferred by the patient. Chemotherapy consisting of cyclophosphamide, vincristine, adriamycin and steroid was started. Patient was given 6 cycles of chemotherapy and was followed up after 6 months. There was no signs of recurrence clinically, radiologically and on haematological picture.
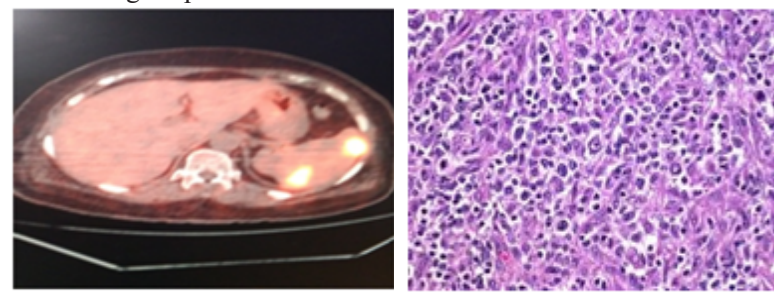

Figure 1: FDG PET Showing Figure 2: Histopathological Increased Uptake In Spleen Examination Showing Primary Splenic Lymphoma 


\section{DISCUSSION}

The nonspecific presentation of Primary Splenic lymphoma creates a real diagnostic predicament for the doctors. Along with Das Gupta et al various other authors have defined PSL. Kraemer et al (6) diagnosed PSL in patients with splenomegaly, cytopenia of at least two hematologic cell lines in the absence of peripheral lymphadenopathy. Kehoe et al (9) defined PSL as NHL arising primarily in the spleen or as NHL principally confined to the spleen and its local lymph nodes. This case report fits into the diagnostic criteria of Kraemer et al and Kehoe etal.

Our patient presented with low grade fever, loss of appetite and abdominal fullness which is consistent with most of the published data $(3)(4)(8)$

PET CT in our case showed FDG uptake only in spleen sparing all other organs which was consistent with the diagnostic criteria of PSL. Hybrid PET/CT has become the standard imaging modality for initial staging, follow-up, and treatment response assessment in patients with lymphoma and has proved superior to CT in these settings.(7) Ultrasound guided trucut biopsy followed by histopathology and immunohistochemistry is an effective and safe way to diagnose a patient as was done in our case.

According to Han B et al, PSL constituted $72 \%$ of the 54 FUO cases presenting with splenomegaly and concluded splenectomy as an effective diagnostic modality for the same. (10) In patients with PSL therapeutic splenectomy is to be done followed by anticoagulation and chemotherapy by CHOP regimen. (10)

We believe this is the first report where PSL was diagnosed using USG guided trucut biopsy and the patient was managed with chemotherapy instead of splenectomy.

This case demonstrates the ease with which PSL can be diagnosed without splenectomy using percutaneous guided biopsy and PET-CT. Queries to whether splenectomy is the sole treatment modality of treatment for PSL arise and the role of adjuvant therapies like chemotherapy and radiotherapy as effective curative agents should be considered. Therefore a deeper understanding in the role of percutaneous guided biopsy, surgery, chemotherapy and radiotherapy in the diagnosis and management of PSL is the need of the hour.

\section{CONCLUSION}

With this case report we arrive at the conclusion that with a nonspecific clinical presentation of Pyrexia of Unknown Origin, one should have a high suspicion to rule out Primary Splenic Lymphoma as a differential. In patients not consenting to splenectomy (such as ours) or declared unfit via PAC, only CHOP regimen can prolong survival upto 6 months with no relapse of lymphoma.

\section{REFERENCES}

(1) Dasgupta T, Coombes B, Brasfield Rd. Primary malignant neoplasms of the spleen. Surg Gynecol Obstet. 1965:120:947-960.

(2) Cavanna L, Artioli F, Vallisa D, et al. Primary lymphoma of the spleen. Report of a case with diagnosis by fine-needle guided biopsy. Haematologica. 1995;80(3):241-243.

(3) Doshi, K., Stanciu, J., Cervantes, J., Rodrigues, L., Gintautas, J., \& Alwani, A. (2008). Splenic non-Hodgkin's lymphoma presenting as recurrent kidney stones -- an "incidentaloma"?. Proceedings of the Western Pharmacology Society, 51, 55-57.

(4) Gobbi, P. G., Grignani, G. E., Pozzetti, U., Bertoloni, D., Pieresca, C., Montagna, G., \& Ascari, E. (1994). Primary splenic lymphoma: does it exist?. Haematologica, 79(3), 286-293.

(5) Sun, P. G., Cheng, B., Wang, J. F., \& He, P. (2017). Fever of unknown origin revealed to be primary splenic lymphoma: Arare case report with review of the literature. Molecular and clinical oncology, 6(2), 177-181. https://doi.org/10.3892/mco.2016.1110.

(6) Kraemer BB, Osborne BM, Butler JJ. Primary splenic presentation of malignant lymphoma and related disorders- a study of 49 cases. Cancer. 1984;54:1606-19. doi: 10.1002/1097-0142(19841015)54:8<1606::AID-CNCR2820540823>3.0.CO;2-5. [PubMed] [CrossRef] [Google Scholar]

(7) Paes, F. M., Kalkanis, D. G., Sideras, P. A., \& Serafini, A. N. (2010). FDG PET/CT of extranodal involvement in non-Hodgkin lymphoma and Hodgkin disease. Radiographics : a review publication of the Radiological Society of North America, Inc, 30(1), 269-291. https://doi.org/10.1148/rg.301095088

(8) Kattepur, A. K., Rohith, S., Shivaswamy, B. S., Babu, R. \& Santhosh, C. S. (2013). Primary splenic lymphoma: a case report. Indian journal of surgical oncology, 4(3), 287-290. https://doi.org/10.1007/s13193-013-0243-x

(9) Kehoe J, Straus DJ. Primary lymphoma of the spleen: Clinical features and outcome after splenectomy. Cancer. 1988;62:1434-8. doi: 10.1002/1097-0142(19881001)62: 7<1433::AID-CNCR2820620731>3.0.CO;2-V. [PubMed] [CrossRef] [Google Scholar]

(10) Han, B., Yang, Z., Yang, T., Gao, W., Sang, X., Zhao, Y., \& Shen, T. (2008). Diagnostic splenectomy in patients with fever of unknown origin and splenomegaly. Acta haematologica, $119(2), 83-88$. https://doi.org/10.1159/000118632 